\title{
The contribution of dermoscopy in Darier's disease
}

\section{Ouiame EL Jouari, Ghita Senhaji, Salim Gallouj, Amina Lamouaffaq, Fatima Zahra Mernissi}

Department of Dermatology and Venereology, University Hospital Hassan II, Fez, Morocco

Corresponding author: Dr. Ouiame EL Jouari, E-mail: eljouariouiame@gmail.com

\begin{abstract}
Darier's disease is a rare genodermatosis. It usually occurs in young people and affects both sexes. It is manifested by yellowish, brown or pink keratotic micropapules, covered with an adherent crust, confluent in layers giving a rough appearance. The dermoscopic aspects of this dermatosis are poorly reported in the literature. We describe the clinical and dermoscopic analysis of the lesions in two patients followed in our department for Darier's disease. We report dermoscopic signs that can help to guide the diagnosis of Darier's disease.
\end{abstract}

Key words: Genodermatosis; Keratotic papules; Folds; Dermoscopy

How to cite this article: EL Jouari O, Senhaji G, Gallouj S, Lamouaffaq A, Mernissi FZ. The contribution of dermoscopy in Darier's disease. Our Dermatol Online. 2019;10(e):e1.1-e1.4.

Submission: 09.10.2018; Acceptance: 12.12.2018

DOI: $10.7241 /$ ourd.2019e.1 


\title{
L'apport de la dermoscopie dans la maladie de Darier
}

\section{Ouiame EL Jouari, Ghita Senhaji, Salim Gallouj, Amina Lamouaffaq, Fatima Zahra Mernissi}

\author{
Department of Dermatology and Venereology, University Hospital Hassan II, Fez, Morocco
}

Corresponding author: Dr. Ouiame EL Jouari, E-mail: eljouariouiame@gmail.com

\section{RÉSUMÉ}

La maladie de Darier est une génodermatose rare. Elle survient généralement chez le sujet jeune et touche les deux sexes. Elle se manifeste par des micropapules kératosiques jaunâtres, brunes ou rosée, recouverte d'une croûte adhérente, confluentes en nappes donnant un aspect rugueux. Les aspects dermoscopiques de cette dermatose sont peu rapportés dans la littérature. Nous décrivons l'analyse cliniques et dermoscopique des lésions chez deux patients suivis dans notre formation pour maladie de Darier. Nous rapportons des signes dermoscopiques pouvant aider à orienter le diagnostic de la maladie de Darier.

Mots clés: Génodermatose; Papules kératosiques; Plis; Dermoscopie.

\section{INTRODUCTION}

La maladie de Darier est une dermatose acantholytique et dyskératosique chronique, liée à des lésions intrinsèques des systèmes d'adhésion interkératinocytaire, a transmission autosomique dominante [1]. Elle se manifeste par des micropapules kératosiques jaunâtres, brunes ou rosée, recouverte d'une croûte adhérente, confluentes en nappes donnant un aspect rugueux [2] . Sa dermoscopie est peu étudiée. Nous rapportons les aspects dermoscopiques retrouvés chez deux patients atteints de maladie de Darier.

\section{OBSERVATIONS}

\section{Cas $\mathrm{N}^{\circ} 1$}

Femme de 61 ans, sans ATCD pathologiques notables, qui présente depuis 3 ans des papules prurigineuses nauséabondes intéressant les plis. L'examen a objectivé des papules kératosiques érythémato-violacées, confluentes en placards à contours irréguliers, en rétro-auriculaire, au niveau des plis du cou, intermammaire, associée à une hyperkératose sous unguéale et trachyonychie (Fig. 1). La dermoscope a mis en évidence la présence de structures polygonales jaunâtres entourées d'un halo clair fin (Fig. 2).

\section{Cas $\mathrm{N}^{\circ} 2$}

Homme de 66 ans, antécédent de cas similaires dans la famille, qui présentait des lésions érythematobrunâtres, prurigineuses et malodorantes disséminées sur le corps, aggravées durant l'été et en cas de transpiration profuse. L'examen avait objectivé des papules brunâtres, hyperkératosiques, profuses réalisant des placards lichenifiés (Fig. 3), des lésions cupuliformes palmaires et des stries longitudinales rouges et blanches des ongles. En dermoscopie on notait la présence de plusieurs structures polygonales, star-like ou ovales, arrondies en forme de zones jaunâtres et parfois brunâtres, de différentes tailles, entourées d'un halo blanchâtre mince (Fig. 4).

Létude anatomo-pathologique de la biopsie cutanée chez les 2 malades révélait au niveau de l'épiderme des zones hyperparakératosique de tailles différentes, une dyskératose avec des corps et grains ronds, et une acantholyse focale avec la formation de fente suprabasale en faveur de la maladie de Darier (Fig. 5). Le traitement proposé était symptomatique.

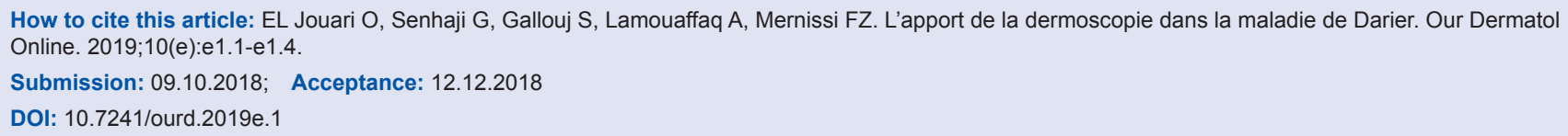



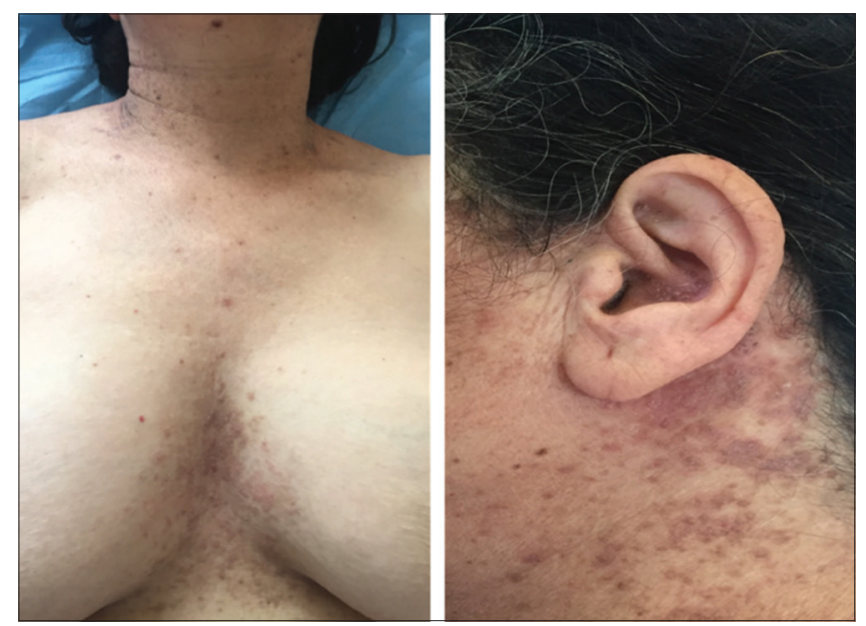

Figure 1: Papules kératosiques érythémato-violacées en rétroauriculaire, au niveau des plis du cou et en intermammaire.

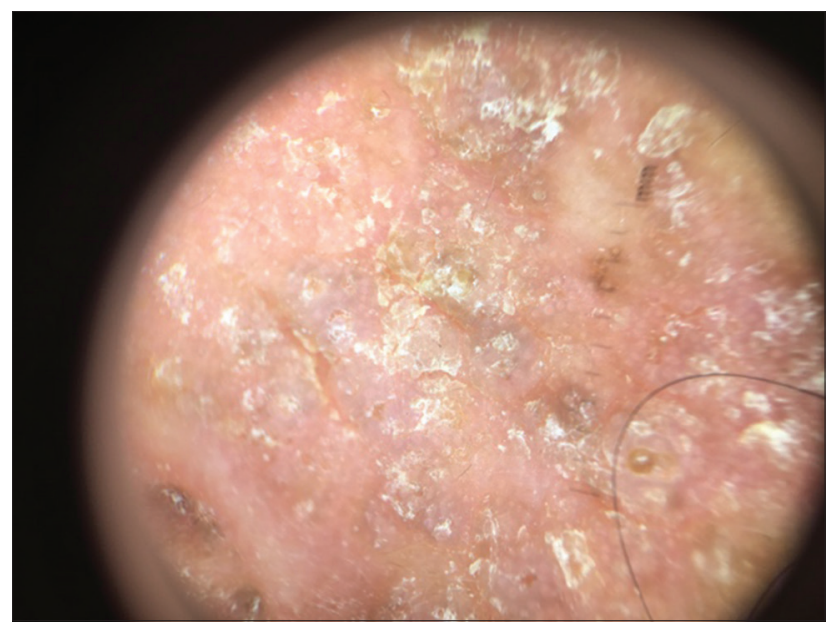

Figure 2: Structures polygonales jaunâtres entourées d'un halo clair fin.

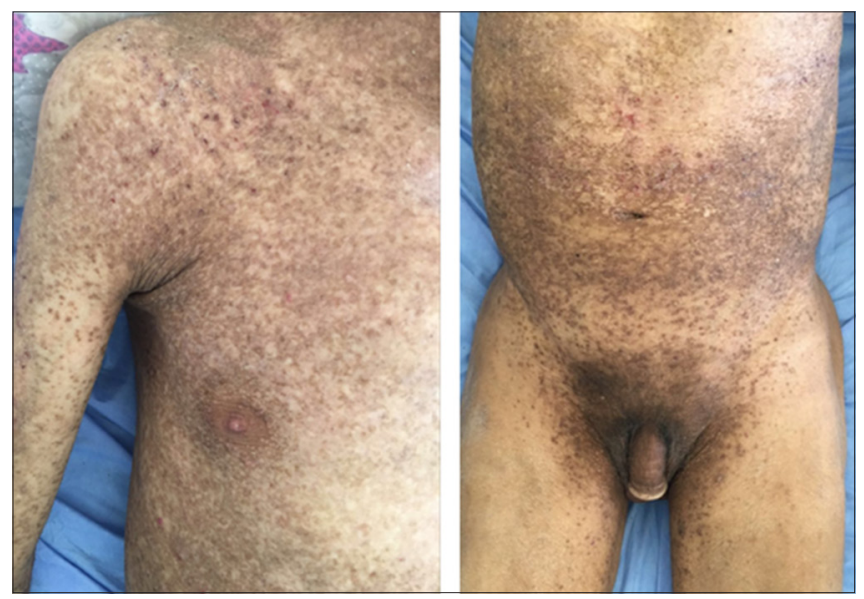

Figure 3: Multiples papules brunâtres hyperkératosiques diffuses.

\section{DISCUSSION}

La maladie de Darier est une maladie héréditaire autosomique rare [3]. Elle est secondaire à des

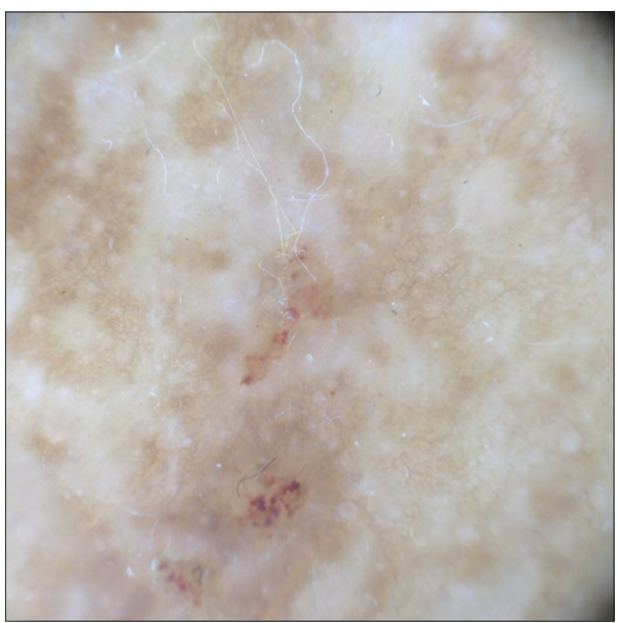

Figure 4: Structures polygonales, star-like ou ovales, arrondies en forme de zones jaunâtres et parfois brunâtres, entourées d'un halo blanchâtre mince.

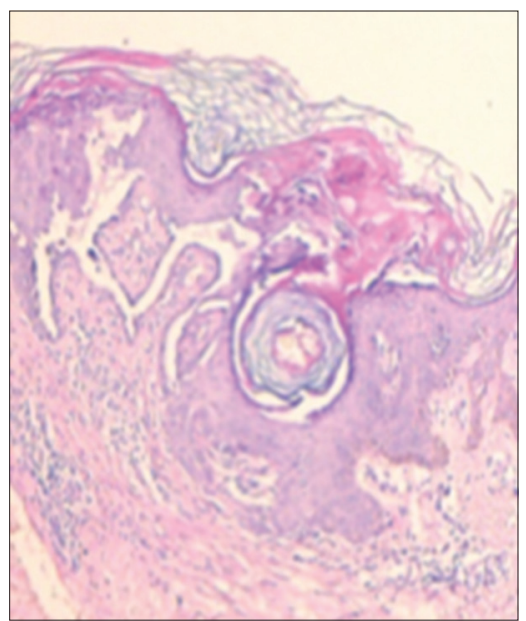

Figure 5: Image histologique (coloration HES, G* 40) montrant une hyperkératose, des fentes suprabasales avec dyskératose acantholytique, et des corps ronds dans la couche granulaire.

mutations du gène ATP2 A2 localisé en 12q, codant pour la voie sécrétoire ça ++ ATPase du réticulum endoplasmique provoquant des changements dans les signaux intracellulaires dépendant du calcium, entraînant une perte d'adhésion cellulaire dans l'épiderme, conduisant à l'acantholyse et l'apoptose [2] Son sex ratio est 1 et aucune différence entre les groupes ethniques n’a été identifiée $[1,3]$. La maladie débute généralement entre 6 et 20 ans, avec un pic au début de la puberté (11-15 ans) [2]. Les lésions primaires sont des papules kératosiques, parfois croûteuses, allant du rouge au brun, qui se développent préférentiellement dans une distribution "séborrhéique " impliquant le tronc, le cuir chevelu, le visage et le cou. Ces lésions ont tendance à devenir confluentes et peuvent former des masses papillomateuses [2]. Peu de données publiées concernant la dermoscopie de cette rare 
entité. Les aspects dermoscopiques décrits sont la présence de plusieurs zones polygonales, étoilées ou arrondies-ovales jaunâtre et brunâtre de différentes tailles, entourées d'un halo blanchâtre fin [4]. Des lésions multiples ont été examinées chez les deux patients révélant les mêmes caractéristiques dermoscopiques. Les principaux aspects histologiques observés au cours de la maladie de darier sont l'acantholyse et la dyskératose. Lacantholyse est due à une perte d'adhérence cellulaire et est responsable des fentes suprabasillaires. La dyskératose correspond à la différenciation prématurée des kératinocytes. Ces cellules dyskératosiques sont décrites comme des "corps ronds" dans la couche malpighienne et des "grains" dans la couche cornée. Lépiderme recouvrant ces cellules dyskératosiques est hyperkératosique [3]. Une corrélation anatomo-clinique a été établi afin de mieux comprendre ces aspects dermoscopiques. En effet, les structures polygonales correspondent histologiquement à l'hyperkératose, tandis que les corps ronds correspondent aux grandes cellules claires de l'épiderme [4]. A travers cette publications, nous soulignons des aspects dermoscopiques d'une dermatose acantholytique rare, dans le but d'aider les cliniciens a mieux identifier cette dermatose et a la différencier des autres troubles acantholytiques touchant les plis. À l'heure actuelle, il n'existe aucun traitement curatif et la rémission à long terme est rare. La prise en charge consiste à éviter les déclencheurs (soleil, macération), appliquer les mesures de photoprotection. Les rétinoïdes oraux constituent le traitement de référence pour les formes étendues. Les traitements physiques sont réservés pour les formes localisées. Tandis que les dermocorticoïdes et les crèmes émollientes kératolytiques avec d'urée ou d'acide lactique peuvent être utilisé pour soulager les symptômes [1].

\section{CONCLUSION}

La dermoscopie dans la maladie de Darier est spécifique et constitue un outil non invasif permettant d'exclure d'autres dermatoses avec un aspect clinique similaire.

\section{Consent}

The examination of the patient was conducted according to the Declaration of Helsinki principles.

\section{RÉFÉRENCES}

1. Valois P-Y, Girault, J-J, Morand. Maladie de Darier. EMC Dermatologie. 2016;11:1-9.

2. Buteică E, Burada F, Stoicescu I, Stănoiu B, Georgescu CV. Darier disease and Hailey-Hailey disease. Rom J Morphol Embryol. 2007;48:423-6.

3. Engin B, Kutlubay Z, Erkan E, Tüzün Y. Darier disease: A fold (intertriginous) dermatosis. Clin Dermatol. 2015;33:448-451.

4. Lacarrubba F, Verz AE, Errichetti E, Stinco G, Micali G. Darier disease: Dermoscopy, confocal microscopy, and histologic correlations J Am Acad Dermatol. 2015;73:e97-9.

Copyright by Ouiame EL Jouari, et al. This is an open-access article distributed under the terms of the Creative Commons Attribution License, which permits unrestricted use, distribution, and reproduction in any medium, provided the original author and source are credited.

Source of Support: Nil, Conflict of Interest: None declared. 